\title{
Téoros
}

Revue de recherche en tourisme

\section{Les parcs humanisés... de troisième génération}

\section{Jean-Claude Jay-Rayon et Brigitte Morneau}

Volume 14, numéro 1, printemps 1995

Les parcs : des réseaux en mutation

URI : https://id.erudit.org/iderudit/1077054ar

DOI : https://doi.org/10.7202/1077054ar

Aller au sommaire du numéro

Éditeur(s)

Université du Québec à Montréal

ISSN

0712-8657 (imprimé)

1923-2705 (numérique)

Découvrir la revue

Citer cet article

Jay-Rayon, J.-C. \& Morneau, B. (1995). Les parcs humanisés... de troisième génération. Téoros, 14(1), 31-36. https://doi.org/10.7202/1077054ar d'utilisation que vous pouvez consulter en ligne.

https://apropos.erudit.org/fr/usagers/politique-dutilisation/ 


\title{
Les parcs humanisés... de troisième génération
}

\author{
Jean-Claude Jay-Rayon et Brigitte Morneau*
}

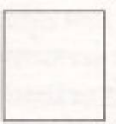

L'bumanité a towjours lutté contre les environnements auxquels elle ne powvait pas s'adapter sur-le-cbawp. fuyant, entre autres, les étendues sauvages quand elle ne les détruisait pas, et cela dans n'importe quelle région du globe.

\section{Cowrtisons la terre René Dubos}

Récapitulons à la manière des gens simples, l'histoire de la naissance des parcs naturels. Et redevenons pour un moment un marcheur solitaire dans le grand-espace nordaméricain, à la redécouverte de la nature et

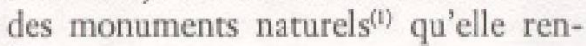
ferme.

Car, c'est bien de cette façon pédestre, que s'est constituée au cours du XVIII et XIX ${ }^{\mathrm{e}}$ siècle, l'idée d'une géographie de la conservation des sites exceptionnels, en opposition avec un monde industriel, brutal et destructeur. Et cela, à une époque où le beau et l'esthétique, le majestueux des paysages, n'étaient pas encore submergés par un jargon écosystémique et biologisant.

Mais on peut aussi faire remonter le concept de préservation au Moyen-Age, et aux forets domaniales ainsi qu'aux territoires de chasse seigneuriaux - ou encore - à l'Antiquité, et à la notion de lieux naturels sacrés habités par les esprits. L'idée n'est donc pas nouvelle, et l'Homme au cours de son évolution a toujours su pratiquer deux tendances: celle de lexploitation-destruction et calle de la préservation à la fois!

Ainsi à une période encore assez récente et craintive on croyait que l'Homme industriel allait détruire tous les milieux naturels. Ceci entraîna du même coup d'autres hommes à s'y opposer, et à prendre position à titre de gardien... d'un xparadis $\%$ en péril: anature pure et sahatrice, à lamanière de Jean-Jacque Roussent.

Monsieur Jean-Claude Jay-Fayon est chercheut concepteur et maitre de conférences. Madame Brigitte Morneau est associée au cabinet-conseil TANGAAM, of se spdoal ise dans la mise envaleur touristique d'environnements naturels etculturels.

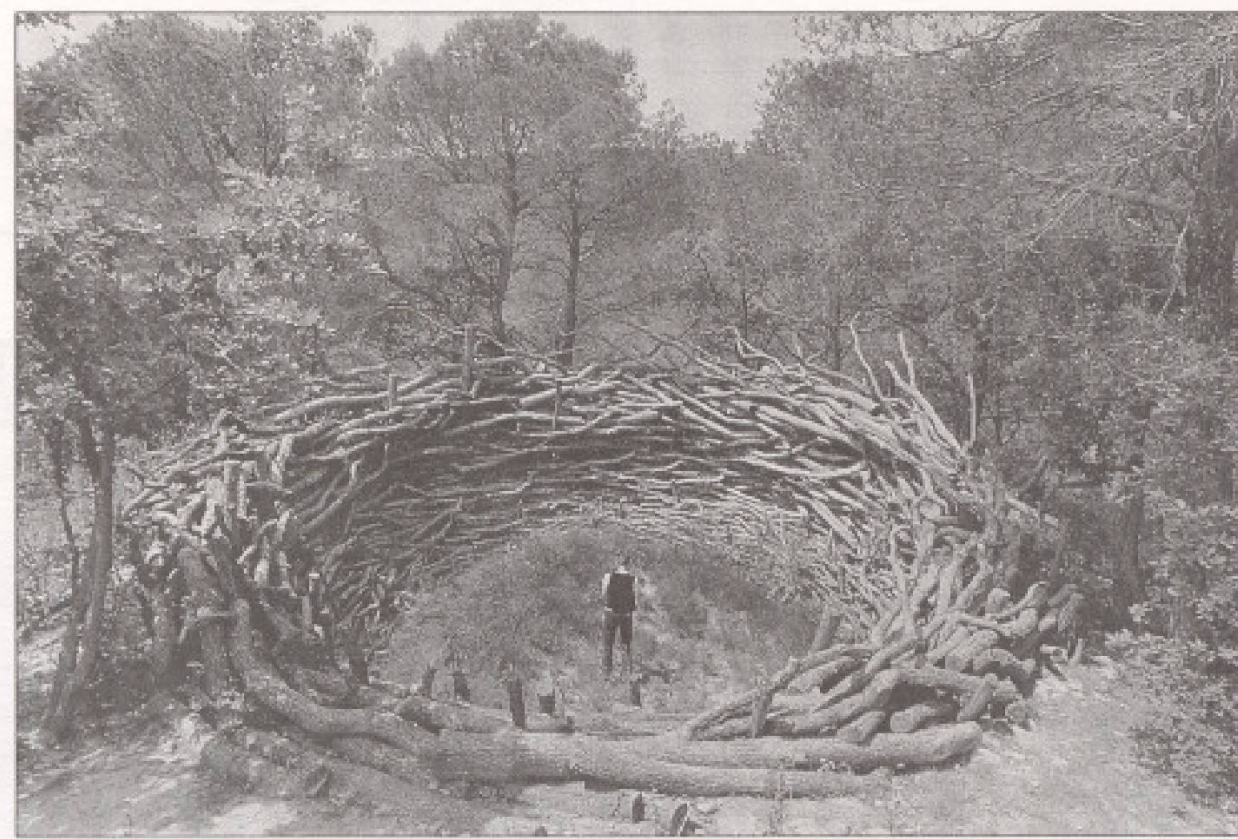

Le nid, Culture-Nature... la nidbe coologique universelle. Sculpture monumentale réalisée en France, dans le prolongement des écoles de pensée, anglo-américaines, reliées au Eartb work, Land art et à colle de la collahuration aih nature. Les forets domaniales et autres, devenues récréotouristiques, et jusqu'ici fardinés par Pentretien des sous- hois, nécessitent dorénavant - pour êtue plus humanisées - une nouvelle approche relevant de l'art environnemental. Ce qui octroie une plus value a la nature. Ce dowt la nature brute des paro

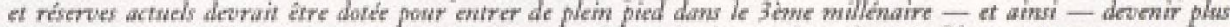

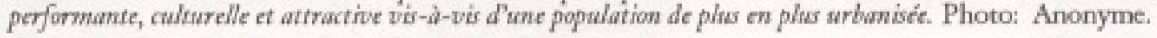

C'est alors quenaquit, à partir d'un constat réel et objectif mais, limité dans l'espace et le temps, la volontéde protéger la faune, la flore etle milieu abiotique, quel'ondéclara en danger, après les avoir considéré euxmêmes dangereux pendant des millénaires. Etrange retournement des perceptions!

Puis, surgit l'idée du sdroit» de la nature sur l'homme, et du adroits des arbres, des montagnes, des rivières... sur l'écologie humaine tout court. ${ }^{1(0)}$ A cette étape, il n'en fallaitpas pluspourdéclencher chezcertains groupes une vocation, suivi d'une mission jugée indispensable à toutes les sociétés modernes, non seulement dans le temps présent, mais aussi pour les générations futures!

Pour ce faire, on passa alors de la démarche biogéophysique initiale à une autre: géopolitique, et on constitua dans de très nombreux endroits des parcs nationauxfédéraux - des parcs nationaux-provin- ciaux - des pares régionaux, locaux, municipaux, de quartier et de voisinage. Et par *prudences, on expropria et «déporta» les familles et les personnes qui y résidaient!

Ainsi surgit au cours des années 19601980, une série de parcs dits naturels, sans homme et sans culture implantée - et aussi - sans grande signification environnementale spectaculaire. C'est à cette même période, qu'au NouveauBrunswick, s'enclencha un procèsqui allait faire date: celui d'un certain 7acky Voutour contre Paro Canada, à Koncbibouguac lequel s'abemina jusqu'aur phus bautes instances judiciaires. Au même moment, et au nom d'une certaine conception du fait naturel, on organisait l'éviction systématique des individus et la destruction de leurs habitats, au parc Forillon, sur l'Tle Bonaventure, etc.

En réalité, la question qui se pose aujourd'hui est la suivante! $Y$ avait-il à l'origine, un seul modèle de développement/aménagementpossible, ouplusieurs? 
L'un, sans écologie humaine, plusou moins «contre l'hommes. Et l'autre, plus humaniste, qui aurait permis aux familles et aux individus - moyennant certaines conditions - de rester en place et de faire partie intégrante des parcs et réserves tels qu'on les connaît aujourd'hui?

La réponse est qu'il y avait deuxoptions et que pour des motifs précis quel'on retrouve énoncés au sein de la «deep ecology», on a privilégié l'une, plutồt que l'autre. Car, il faut maintenant en convenir, des parcs et réserves mondialement connus ont su respecter une démarche intégratrice où les écologies humaines, animales et végétales font encore bon ménage.

Àce sujet, il s'agit de mentionner le parc de Kakadu en Australie qui a maintenu en son sein les Aborigènes dans leur milieu de vie. L'espace de Gordes, Roussillon... et du village des Bories, dans le sud de la France, qui a inclu les villages et les terres cultivées environnantes. La réserve africaine du Masai-Mara en Tanzanie, dans la plaine du Sérengeti, qui a inclu les Masais, etc. Darss cet ordre d'idée, n'aurait-il pas été smubaitable de reppecter l'implantation ancestrale des gens de la Gaspésie, de ceux de la Cöte-Nord, du Saguenty, duLacSaint-7ean... quitteà negracier un nouveau wcontrat environnementals avec ent?

\section{Plus de parcs... oui bien sûr! Mais où ça?}

L'actuel déficit d'espaces conservés et maintenus dans leur biodiversite ${ }^{\text {(b) }}$ - ce à quoi nous souscrivons en général mais, avec des nuances - devrait se compléter par la préservation de nouveaux lieux mais, cette fois, situés ailleurs que dans la grande nature et la forêt. Aillewrs que dans une nature plus encline à abriter une certaine primitivité, qu'à accueillir une sociéte de phus en phus urbanisée, en quête de paysages bicoligues, fagonnés par des sieddes de culture rurale.

Notre raisonnement repose sur nos origines et l'environnement dont nous sommes encore imprégnés. Au risque de surprendre, nous sommes les héritiers et le produit d'écosystèmes qui n'ont presque rien à voir avec la forêt primordiale et une nature brute et complexe sur le plan locomoteur. Plus précisément, la nature sauvage de nombreux parcs et réserves n'a rien d'attractive en ce qui a trait à notre système de cognition-perception et de locomotion. En conséquence, il ne faut pas s'étomer que la participation y soit faible.
Nous sommes en réalité le produit d'une grande prairie odorante, jadis située en Afrique orientale, enveloppée d'un climat subtropical et parcourue d'alizés, avec un relief constitué de collines douces et parsemées de bosquets, aboutissant à des rivages... Le jardin $d$ Arcadie, si cher au romantisme du XIX* siècle.

En fait, et même si nous pouvons occuper temporairement presque n'importe quel milieu sauvage ou artificiel, notre smémoires bio-culturelle ramène la majorité d'entre nous sur les terrains de l'humanisation. Ceux de nos campagnes en particulier! Et c'sot ausi pour cos raisons fondamentales que nous apprécions particulièrenent l'Estrie et le Vermont - les rivages d'Acadie, le ajardin de Fronce et d'Italiew, la campagne anglase, at irlandase..., berwowp phus que n'importe quel sin lands, sombre et browsailleax.

Le deuxième aspect est essentiellement culturel, et s'appuie sur des écritrures aussi bienoccidentales qu'orientales. A ceniveau on ne trouve que peu de traces historiques incitant les sociétés modernes ou anciennes à effectuer des *investissements* massifs dans des parcs et réserves naturels, forestiers, montagneux, marécageux, désertiques...

Qu'est-il dit de ces milieux naturels, sons culture depuis la nuit des temps? "Disséminés dans les forêts primitives [...] les descendants de Noë perdirent [...] leur humanité de génération en génération, et devinrent des créatures solitaires et infîmes, vivant sous un toit de branches et de feuilles Brutes ... Bruteaux, inquiets, incestueux, ils ne connaissaient pas de Loi supérieure.... (Les Géants de Vico,p. 21). Ne croit-on pas voir là quelques similitudes avec $\mathbf{L a}$ Bête lumineuse de Perreault, ou le film américain Délivrance?

Plus proche de notre époque, on peut aussi lire: *Cet être bestral qui vit seul dans la forêt, nu et hirsute, fort et agressif [...] se nourrissant d'herbe et de chair de gibier [...] On l'appelle au Moyen-Age l'homme sauvage [...]. On le retrouve bien plus tard souslestraitsde Tarzan... $\gg$ (L'aventure du chevalier, p. 105).

Le troisième argument concerne directement le Québec rural et les terres agraires abandonnées. Actuellement, on compte environ plus de 3000000 hectares qui retournent en friches et déparent l'environ- nement en bordure des routes. Là ou demeurent $80 \%$ de la population.

Ce phénomène porte déjà lourdement atteinteà la qualité paysagère de nombreuses régions, et ralentira le développemenț touristique deces dernières dans le futur. $\dot{A}$ l'avenir ce sont surtout cer zomer qui devraient être conservées et protégées, comme le font déà la Suiser, I'Angleterre, la France et certains Etats de la Nowvelle-Angleterre, en favoricant whe agriculfure de paysige. Concept très peu connu au Québec.

À l'aube de l'an 2000, des parcs et réserves ruraux, pastorauxet maritimes pourraient êtreconstituéssur une base plus humanisée et culturelle. Ces parcs et rếserves de troisième génération permettraient de ralentir le dépeuplement de certaines zones rurales et maintiendraient la qualité du paysage agraire dans des endroits aux caractéristiques esthétiques exceptionnelles. Enfin, ils permettraient la création d'emplois nouveaux, axés sur l'agriculture de paysage.

\section{La locomotion... de l'activité physique, à la perception et connaissance des lieux}

Ả la même période oủ les biologistes effectuaient l'évaluation des écosystèmes pour le zonage géographique des parcs et réserves, et où d'autres personnes déterminaient ce qu'était ou n'était pas naturel; un corps professionnel s'activait : celai des éducateurs plysiques.

Il faut se replonger au coeur des années 1970-1980 pour bien se rendre compte de l'influence de l'activité physique, alors synonyme de santé, de longévité et de bonheur. C'était l'époque où l'acquisition d'une endurance et d'une résistance physique menait en quelque sorte à une forme de... transcendance!

Bref, la plupart des parcs actuels sont encore dessinés suivant des principes de déplacements linéaires et de niveaux de difficulté issus du sport, sans grande considération du fait que la société a mûri, et qu'aux environs de 2006 , elle aura vieilli.

Déjà en 1986, dans le bulletin d'analyse critiquedel'éducation physique québécoise: Intracom, de l'Université de Sherbrooke, nous faisions remarquer que la grande majoritédes sentiersnetenaient pas compte de façon paradoxale, d'une locomotion 
naturelle. En nous appuyantsur lesrecherches de Konrad Lorenz en éthologie humaine nous répéterons donc que: «L'homme qui explore naturellement un territoire procède par "séquences" multiples, avançant, reculant, allant à droite et à gauche, cherchant continuellement le lieu le plus favorable. Lorsqu'ill'a découvert, il a tendance à s'y arrêter, c'est-à-dire à supprimer le mouvement corporel et à s'immobiliser. L'homme n'est actif que par nécessité réelle, sinon c'est un... contemplatif. Ce processus naturel est désigné sous le nom de clino-kinésis et il s'illustre aussi bien au travers du comportement des ruminants en train de paitre ou d'homme en train de chercher des champignons» (Korad Lorenz, 1973).

En somme, il s'agirait pour rendre plus accessible les parcs et réserves, de traduire par un aménagement locomoteur adéquat, le comportement naturel du déplacement humain - et ce-en le rendant plus facile à toute une société.

Sur le plan de l'aménagement global, l'ensemble des circuits devrait converger vers les endroits les plus beaux et les plus significants. Les réseaux de circulation devraient être épurés et donner acoès à des «ooquilles environnementales spectaculaires" axées sur la perception-cognitive, le tout favorisant une attitude contemplative.

\section{De la nature... avec des traces de culture}

Comme le précise Luc Ferry dans le nouvel ordre écologique, au chapitre: *Les ombres de la Terrew: ala nature est belle quand elle imite l'art [et] [...] le souci de reconnaittre et de préserver ce qui paraît déjà humain en elle [...] rejoint ainsi les idées qui nous sont les plus chères: liberté, beauté, finalitén (pp. 261-262).

En nous référantà ce principe, qui d'ailleurs est partagé par une partie de l'éthologie et del'écologie humaines et certains courants artistiques, nous devons admettre que l'Hommenevoitlemilieu naturel que sous un angle anthropomorphique et surtout culturel. En conséquence, on ne peut que souhaiter une abondante présence de tratces culturelles dans les parcs et réserves. Des margues oncriter qui augmenteraich le sentiment dappartenance bumain at sacial: une attitude redberdbé vis-à-vis dlane mature à conserver.
C'est cetteidéequia été d'ailleurs exploitée dans l'Etat américain du Dakota nord, au travers de la représentation des figures présidentielles génntes. Un endroit assez «médiocres à l'origine, où on a su concrétiser un besoin perceptuel, tout en s'inspirant de l'art monumental de la vallée duNil, etdel'histoire politicueaméricaine. Unesynthèseinnovatricequin'a rienà voir avec l'univers Disney, comme oertains se plaisent à le colporter!

En regard de ce que nous venons de dire, dans un parc comme celui de la JacquesCartier, n'y aurait-il pas alors lieu de graver à même certains rochers, l'épopée des Jésuites? Et à cela, ne pourrait-on pas y ajouter la reconstitution d'un site archéologique - la mise en évidence d'endroits évocateurs primitifs - la reproduction de pétroglyphes - les traces des campements de bûcherons....?

Ces dynamiques artistiques que l'on retrouve en Europe aussi bien qu'aux EtatsUnis sont encore peu connus ici. Dénommés *collaboration with natures, «Earth works, ou Land arts, elles s'intègrent en général parfaitement à l'environnementnaturel, pour peuqu'on en contrôle la réalisation.

De plus, et au-delà de l'oeuvre tlle-même, cela procurerait une image unique et spectaculareà certains parcs et réserves; ce qui compenserait pour les qualités très moyennes ou faibles de la nature végétale et animale de certains.

Dans le même élan, cela permettrait aussi d'ouvrir des crénenux novateurs pour les emplois régionaux $x_{\xi}$ particulièrement pour certains artistes, et faciliterait le renforcement des identirés culturelles régionales à des fins touristiques. $\mathrm{Ce}^{\prime} \mathrm{q}^{m i}$ crearuit du futur!

\section{La grande dispersion des} infrastructures et le manque de rentabilité périphérique ou... l'économie environnementale en panne technique

Dans l'ensemble, les parcs et réserves se plaignent - à juste titre d'ailleurs - d'un manque d'achalandage réparti sur l'année. Ex en conséquence - d'uncertain manque de revenus et d'autofinancement directs et indirects reliés à la pratique desactivités de plein air. Cette faiblesse touche directement l'économie locale environante $e^{i t}$.
Quant aux revenus périphériques - ceux qui devraient se situer à la frontière immédiatedela couronne des parcs et réserves -ils sont inexistants, ou presque. Surtout si on les compare avec ceux des parcs américains.

En ce sens, on ne retrouve presque nulle partces $\&$ Factory Outles $\approx$ qui regorgent de matériel et de littérature de plein air au prix du gros. Et qui s'inscrivent comme un renforcement commercial normal dans un cadre environnemental propice à ce genre de consommation.

Ceci est regrettable parce que l'économie du *patrimoine- environnement» dépend surtout des produits dérivés et non pas de l'augmentation d'un prix d'entrée, ou de l'augmentation improbable dessubventions gouvernementales. Ce sont donc de nouveaux montages financiers locaux et régionaux qu'il faut mettre en place si l'on veut financer l'environnement récréotouristique des années 2000+. Celui des parcs et réserves y compris.

Un autre motif - et non le moindre - de s'inquiéter de la sous-rentabilité des parcs et réserves réside dans la grande dispersion des infrastructures de plein air pour desservir une masse de clients réels et potentiels issez modeste. Celle du Québec et de ses visiteurs.

En ce sens, les trente dernières années ont vu se concrétiser la dispersion géographique des infrastructures de service sur la base d'une qualité environnementale qui n'est pas toujours significative. Certains laissent à désirer et sont sans grand attrait récréotouristique compte tenu du coût des aménagements et bâtiments qui s'y trouvent implantés ${ }^{(5)}$,

Les pares, les réserves, les bases de plein air, lescamps et colonies devacances, lescentres de plein air et de jour, etc, se font tous dorénavant une féroce concurrence, sur un immense territoire, et dans un contexte de rationalisation économique, par concentration.

En conséquence, l'économie environnementale toute entière, des parcs et réserves est interpellée, et personne ni aucun endroit n'échappeàcettedynamique. Dans ce contexte, il ne serait alors pas étonnant de constater la nécessité de *fermer $\approx$ du moins temporarement de grands espaces 


\section{CHAINE ENVIRONNEMENTALE Ȧ CINQ UNTTÉS}

Premicre anite emiromementale: L'école (1), le voisinage (2), le quartier (3) et la périphérie urbaine (4) qui se trouvent complétés par des environnements semi-naturels extérieurs. $80 \%$ et plus en Amérique du Nord.

Deuxieme wonite cnvironnementale: La ferme et le village agraires (5) complétés par les terres dumême nomou encore... un environnement sbucoliquem humanise. Environ $8 \%$ en Amérique du Nord et $14 \%$ \ $16 \%$ en Europe.

Troisieme unite ewowonnementale: Lecentre de plein air et la pourvoirie (6), la base de plein (7) air ainsi que les pares régionaux, provinciaux et nationaux (8) qui devraient normalement être accompagnés d'un type d'environnement bátibien déteminé. Environ $4 \%$ is $\%$ d'occupation.

Quatriàne unité entinonnementale: La réserve Écologique (9) et le refuge faunique (10) où l'environnement extérieur naturel prime, $2 \%$ d'occupation environ.

Conquithe anité eworonmenewtale: La nature primordiale (11) propre aux expéditions et à acces tres limité. Tris faible pourcentage d'utilisation de l'ordre de $0_{2}, 01 \%$ environ.

naturels et d'en ouvrir d'autres plus proches des marchés participatifs.

Un des moyens efficaces de renforcer l'attraitenvironnemental d'une région serait de sélectionner les plus beaux sitesetdeconcentrer lesinfrastructures actuelles àl'intérieur et au pourtour de ceux-ci, quitte à les déménager. Cela concerne les parcs et réserves les uns par rapport aux autres, et les espaces à l'intérieur de ceux-ci.

\section{Des parcs et réserves partout, n'importe où... ou dans une logique spatiale!}

Dans un vaste contexte spatial, l'ensemble desespaces «verts-bleusw - dont filit partie les parcs et les réserves - semble peu répondre à une répartition logique des environnements. Du moins, ils ne répondent pas à une logique du rendement écologique, esthétique, éducatif... comme les espaces agraires ou miniers le font en exploitant des terres arables ou les gisements les plus productifs, pour se maintenir dans des marchés compétitif́s. Ceci est même

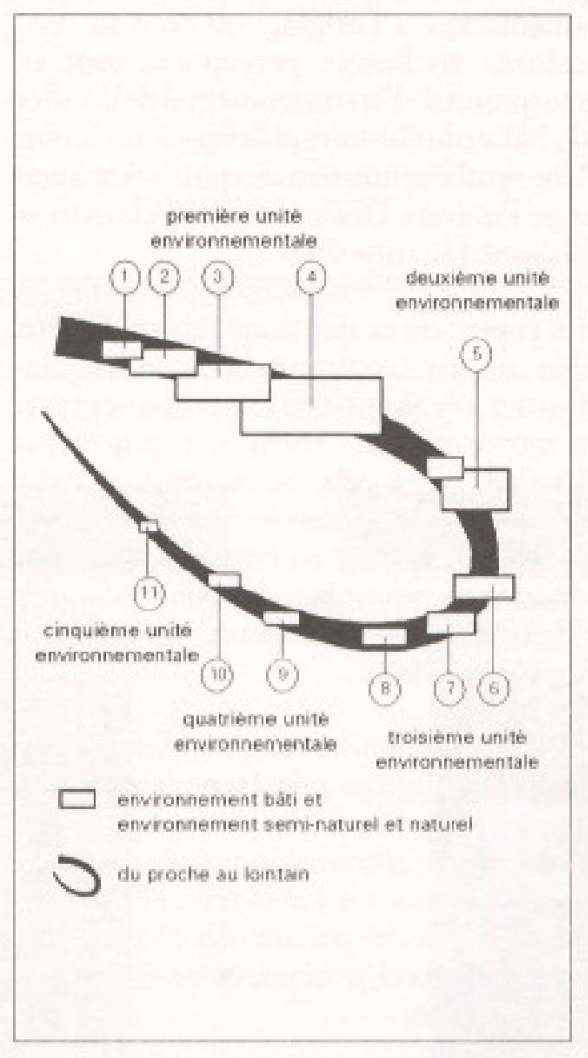

une notion encore rejetée ou méconnue de la plupart des écologistes environnementalistes des annés $1990-2000$ !

En conséquence, les espaces *verts-bleus* se situent difficilement au sein d'une hiérarchie de l'offre et de la demande récréative, touristique et ducative. Ils agissent même parfois en duplication les uns par rapport aux autres, indiquant ici et là en région, des surplus ou des pénuries.

En fait, les espaces averts-bleuss se présentent rarement sous la forme d'une schaine environnementales colhérente qui s'inscrirait de façon harmonieuse au sein de l'aménagement global du territoire québécois. Jusquấa maintenant, ils permettent peu d'offrir une gamune de sites complémentaires à la sociéré. Mais en fait, répondent-ils aux besoins comportementaux naturels de la majorité? Car, comme l'exprime René Dubos: *L'histoire complexe de notre espece fait que presque chaque être humain aspire à retourner de temps à autre à chacune des différentes expériences del 'évolution, celle du paléolithique [...] celle du fermier et de l'éleveur, celle du citadin» (Courtisons la terre).

Une réponse affirmative serait ma heureusement difficile à formuler, surtout dans un contexte d'urbanisation grandissante ou il $y$ a perte continue $\approx$ d'espace - temps*. Ce qui devrait amener les parcs - quels qu'ils soient - à procéder de la cour d'école, jusqu'aux territoires asauvages», propres au tourisme d'aventure ou d'expédition ${ }^{(6)}$.

Ce questionnement en profondeur nous avait déjà été posé dès la fin des années 1980. A cette époque, nous avions été invités à participer à un raisonnement d'envergure internationale concernant l'aménagement global des sites de plein air au Québec, en France et en Belgique. Par la saite, avatit été pwblé un livre de référence préface par mudame Gro Harlem Brundtland, Premier ministre de la Norvege; la personne mêne ruianaitprésidéla Commissonmondade sar liemviromenent et le développentent de 1984 à 1987, pour lONU, en compagnie de René Dubos.

Qu'en seraient-ils donc de la démarche spatio-temporelle envisagée, et des résultats anticipés, si on acceptait au Québec et ailleurs - d'appliquer une cohérence d'ensemble à la répartition des espaces werts-bleus»? Et ce, de façon à mieux répondre aux besoins de cę début du troisième millénaire. Ce qui nécessairement resituerait les parcs et réserves actuels dans un nouveau contexte géographique et social.

Cette achaîne environnementale à cinq unités", sans être exhaustive, a été élaborée en tenant compte des énoncés de l'UNESCOet de l'ONU, recommandant de conserver et de préserver, mais aussi d'offrir aux générations futures, des environnements progressifs et complémentaires, à partir des masses humaines urbanisées. Là où réside la majorité de la population!

Nous avons cru nécessaire d'octroyer, dans chaque unité, une identité spécifique ( 1 à 11) a chacun des lieux, de façon à ce que le "modèles coincide avec la réalité environnementale québécoise. Au cours de cette recherche appliquée, nous nous sommes aperçus que les environnements $1,2,3,4$ et 5 étaient souvent négligés (pénurie). Et quel'on avait tenté en général de privilégier au cours des années 1960/ 1985 - de façon plus ou moins «artifi- 
cielles et paradoxale, les espaces $6,7,8,9$, 10 et 11 (surplus). Nous en avons alors déduit - toutes nuances mises à part que lors des *Golden decades $*$, sur une période d'au moins vingt-cing ans, on avait favorisé une approche environnementale de sfuites, de sretour aux sourcess, de "grande natures, et de parcs et réserves naturels éloignés. Àl'inverse, pendant que l'on investissaità la manière de Jean-Jacques Rousseau, loin des villes, ces dernières ne cessaient d'accuser des déficits, d'espaces * verts - bleusw, et de volumes de vie tout court. De quoi susciter la réflexion des décideurs de l'an 2000/2010!

Deplus, cette démarche environnementale plutôt «passéistes, et basée sur l'utopie d'une «vraienatures à recouvrer, a accentué une ruptureconceptuelle et concrète entre les milieux urbains, ruraux et primordiaux. Avec comme résul tat: l'oulli del lenvironnement charnière semi-naturel et agro-forestier. Celui qui, justement correspond le mieuxà notre «mémoire génétique» et historique: bio-culturelle.

À ce raisonnement il s'agit d'ajouter que la *chaîne environnementale* telle que structurée se scinde elle-même en cinq grandes unités distinctes. Cette proposition rationnelle correspondant à la fois a ux comportements fondamentaux de l'Homme - à ses capacités relatives d'adaptation en territoires éloignés - et aussi - à une économie financière et à une éthique, quant aux investissements publics à effectuer proche des gens, pour que ces espaces soient réellement accessibles à la majorité d'une société.

\section{Parcs et réserves... \\ $2000 / 2010$ en perspective}

Alors que la première et deuxième générations des pares étaient surtout axées majoritairement sur la préservation des monuments naturels, curiosités naturelles et écosystèmes rares; la troisième pourrait être différente et venir se situer en complémentarité.

Elle pourrait carrespondre à la tendance progresiste du comportement bumain et aborder l'evolution port-industrielle, en offrant une whyper-natures sur mestar, ou wne atednonatures bien intégrét?

Alors que jusqu'à présent la démarche passéiste tendant à surprotéger le milieu naturel dans son état premier; il est probable que dans les années à venir, la sociétés'y réassociera pour l'améliorer, lemodi fier, et le prolonger. On voudra startout le rendre plas performant et plas spentaculaire aux ni-

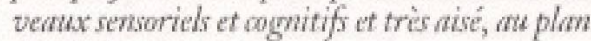
des deplacements internes.

En fait, ils'agit d'évaluer la période actuelle comme une sorted intervalle plusou moins située entre une vision conservatrice qui se désagrège au fur et à mesure des multiples privatisations. Et une autre, résolument avant-gardiste, qui veut que les achoses bougents et que l'on innove, de façon à créer de nouvelles entreprises et des emplois. Cequi est indispensable aux localités périphériques des parcs et réserves.

Pour ce faire, il est dès maintenant indispensable de mettre de l'avant une nouvelle ingénierie environnementalequi modifiera à certains endroits la nature elle-mêtne et ce, pour le mieux desperceptions. Il pourrait s'agir entre autre de la volonté de se servir de plusieurs écosystèmes en place, et de les «surdimensionners pour les rendre plus attirants. Ou encore, d'introduire des infrastructures légères et lourdes, comme des chemins et routes panotamiques, des funiculaires intégrés, des ascenseurs transparents, des éclairages au laser - ou bien - de favoriser la semi-domestication de la faune, etc.

Car inst bien vers od que l"on se dirige quand an constate à titre dearmples le sactes de "Cadillac roads à Acadia powk Lans le Maine,

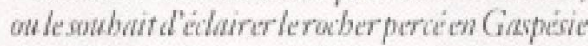
(malbearasement rojeré), on chore, les promenades nootoriseres wer le salle fin da parc de St-Auguatime, en Floride nord...

Après tout, les parcset réservesétant financés par les impôtsde toute une société, il n'y a aucune raison juridique ou logique pour laguelle on favoriserait une forme de pratique environnementale par rapport à une autre; pour autant que toutes respectent le milieu et ses capacités de support et de regénération.

Si nousavançons I'hypothèse d'une ahypernatures et d'une etechno-natures avancées, c'est que - entre autre chose - les parcs et réserves actuels se situent dorénavant en compétition avec des infrastructures comme siosphere Twow, le «Biodômes... De plus, ils font face à la montée fulgurante et massive des communications visuelles, dont la plupart présentent le milieu naturel sous une facette du splus que natures.
Le fait est que des émissions comme *Travel, travelw présentent à des millions de spectateurs des plus beaux paysages récréotouristiques au monde. Les cinémas *Imax influencentdes milliers de familles et montrent la nature sous des aspects plongeants et périphériques à couper le souffle! Du jamais vu! Quant aux magazines géographiques, dont le plus célèbre National Geographic, ils sélectionnent les photos les plus exceptionnelles et inusitees. Devant tout ceci, que vandront dans les dix amées à venir certains aninagements approximatifs au sein des paro et réerves?

Quelques personnes pourraientarguer que rien nevaut une situation réelleet naturelle! Faux en partie, répondrons-nous, car le cerveauhumain, est dèsaujourd'huicapable d'être mieux servi en *perceptions naturelles» par... I'artificiel; et très bientôt, par le virtuel.

Alors que faire? Le plus rapidement possible, replacer une grande partie des parcs et réserves dans un contexte évolutif. Ou encore, accepter le fait que certains apparaissent de plus en plus sous l'angle de quelques reliquats du monde naturel; lesquels seront visités par une quantité de moins en moins grande d'individus. Un univers à côté duquel se développera des milieuxsemi-naturels, satisfaisantsl'écologie humaine a priori. Autrement dit, des jardins semi-naturels, des campagnes fleuries, des boisés jardinés, qui, de façon symbolique, se rapprocheront de plus en plus des mythes heureux de l'Arcadie! Le parc des chutes Montmorency, proche de Québec, préfigure déja ce nouveau type d'environnement à haute fréquentation populaire malgré certaines erreurs d'aménagement notoires.

Dans la mesure où les parcs et réserves devront répondre de plusen plusà une demande de nature spectaculaire, et augmenter la gammede leurs clientè̉les, il semble nécessaire qu'ils améliorent la performance naturelle, perceptuelle et cognitivedeleur milieu. En somme, il s'agira d'ajouter une valeur supplémentaire à la nature sauvage, par l'amélioration des écosystèmes. Les moyens techniques et mécaniques d'accès devant y occuper aussi une place importante. En somme, le concept de valeur ajoutée et de qualité totale concerne aussi la nature offerte au public. 


\section{Conclusion}

Que l'on ne s'y méprenne pas, nous sommes convaincus qu'il faut conserver et préserver des sites exceptionnels et les protéger contre un certain vandalisme, ou une exploitation destructrice. Nous aimons la nature mais, sans trop vouloir la 4materners, ou vouloir systématiquement la maintenir ou la ramener à son état passé. Ce qui est à la fois illusoire et... un peu prétentieux! Nous nous situons comme des environnementalistes progressistes et culturalistes. Notre analyse n'est donc pas une critique. Elle se veut surtout une rééquilibration du type d'utilisation trop exclusive et limitative que l'on fait encore des pars et réserves et de l'environnement en général.

Ànotreavis, rien ne sertplus des'atermoyer sur la question des principes reliés à la privatisation des activités de plein air, des plans de promotion, de protection, de restauration... La question n'est plas li, dans La mesure oul l'environnement et maintenu en équilibre entre une exploitation-destruction inévitable - l'mpactnégatif - etunecapacité de regénération supérieure. Etrien ne prouve en ce domaine que le privé soit moins compétent et consciencieux que le secteur public. Faire des procès d'intention, ne mène nulle part!

De plus, qu'une série d'emplois secondaires dans les parcs et réserves - jusqu'ici soutenus jusqu'à l'extrềme limite du raisonnable, par une société en difficulté économique - disparaissent des listes de paie gouvernementales, n'a qu'une importance relative. Hormis la situation personnelle d'individus, à laquelle il faut compatir, bien sûr! Que le fardin de Métis ${ }^{(19)}$ retourne à une société privée responsable, n'estque mieux, et un juste retour des choses. L'Etat ne s'étant jamais situé à l'origine de la création de ce type d'environnement, on ne voit pas pourquoi il s'y incrusterait!

La réalité, on l'aura sans doute compris, c'est que les parcs et réserves doivent évoluer vers de nouveaux types d'exploitation de leur propre environnement; lesquels généreront de nouvelles entreprises PME et TPE. Lesquelles aussi, créeront du travail, pour de nouveaux marchés précis. Et dans cette optique, il est clair que le produit actuel «natures est tropétriquéou mal ajusté aux besoins de l'an 2000/2010.
En un sens, avoir pensé un instant que l'ćcologiepouvaitse passer d'une économie environnementale, était tout simplement une utopie de plus, insoutenable à moyen terme. L'buwanité a towjours troqué et monnayé toutes les productions de la Terre pour troluer. Et les valeurs esthétique, récréative et cognitive des parcs et réserves n'échappent pas à cet état de fait. Il fallait qu' on y revienne un jour oul'autre!

Voltaire, avecson vert langage disait un jour

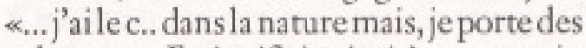
culottes\%. I signifiait ainsi à un certain Jean-Jacques Rousseau, qu'il ne s'agit pas seulement de faire appel à la spensée magique naturelle» pour influencer les choses mais, qu'il faut aussi y ajouter une dose substantielle de nouvelles connaissances - ét done - de culture pour réussir. Les culottes!

Nous insistons à nouveau en répétant qu'il en est de même pour les parcs et réserves actuels, ces lieux en général de «trop grande natures auxquels il s'agirait d'adjoindre le plus tôt possible de sérieux éléments de culture. Et ce, pour qu'ils puissent être mieux reliés à la nature humaine. C'est le concept même de la wnature humanisées, pour la majorité d'entre nous.

En conséfquence, nous arons choisi un angle different et nous nous sommes placó parmi les demandeurs de nowvenutés Nature - $\mathrm{Cul}$ ture. Cenx qui se sentent aspirés par le futur, repousent los stam quo, et ne prennent rien pour acyuis!

Après tout, sinosancêtresdu Nerndertal, ont été capables d'intếgrer avec art et intelligenceles peintures rupestres à la naturedes cavernes; il y a lieu d'espérer que les nouveaux administrateurs, biologistes, récréologues et environnementalistes à venir, seront à même d'en faire autant, en réhumanisant entre autre la nature des parcs et rếserves et en n'éliminant pas la possibilité de consommer un cake comme cela se produisait aux alentours des années $1970-19800^{(0)}+$

\section{BIBLIOGRAPHE RESTREINTE}

BAFNIER, Michel, L'enwironnement, Monuments historiques - Paysages, no 192, avni 1994, pp. 8 . 12.

DUBOS, René, Courtisons la terre, Éditions Stock, 1980, $243 \mathrm{p}$

EIBL-EIBESFELDT, Irenäus, Ethologie, blologie du comportement, Editions scientifiques Naturalia et Biologica, 3eme édition, $1984,748 \mathrm{p}$.

EIBL-EIBESFELDT, Irenäus, L'homme programmd, I'lnnú facteur dóter minant du comportement humain. Flammarion, 1976, $256 \mathrm{p}$.
FALQUE, Max, MILLIERE, Guy et collaborateurs, Écologie et libertí, une autre approche de Penvironnement, LIBERALIA, Economie et liberté, 1992, $378 \mathrm{p}$.

FERAY, Luc, Le nouvel ordre beologique, V'arbre, Fanimal et P'homme, Editions Grasset, 1992, 269 P.

GOUNERNEMENT DU CANADA, Principes directeurs et politiques da geation, ministère du Patrimone canadien, $1994,127 \mathrm{p}$.

GOUVERNEMENT DU CANADA, Rapport statistique 1993 - Rugion du Qubbec (Parce nationaux. Lieux historiques nationaux, Canaux histor: ques nationaux), ministéfe du Patrimoine canadien, $1994,103 \mathrm{p}$.

GOUVERNEMENT DU QUEBEC, La nature en hórtage - Plan d'action sur les parca, ministère du Loisir, de la Chasse et de la Pèche, 1992, 22 p.

GOUVEANEMENT DU OUEBEC, Les parce quabdecols - 1. La politique, ministére du Loisir, de la Chasse et de la Peche, $1992,70 \mathrm{p}$.

HARRISON, Pobert, Forbts, essal sur rimaginaire occidental Editions Flammarion, 1992, $389 \mathrm{p}$.

INRS-URBAINISATION, Qú́bec 2000*, une prospective du Cuábec a l'aube d'un autro sibcle, Chapitres: Le Quebec toujours aux prises avec sa démographie et Le Quebeci une société et une culture sous tension, 1994, pp, 81-108 et 309340

JAY-RAYON, JoanClaude, Bio-mociologie ot bdueation physique... ou le ndcasasire ratour aux sources ot à liessential comportemental, Intracom, bulletin d'analyse critique de l'bducation physique, Université de Sherbrooke, no 16. $1986,4 \mathrm{p}$.

JAY-RAYON, Jean-Claude, Principes de déplacements plein air, Schémas, 1986, 12 illustrations.

JAY-AAYON, Jean-Claude, Plein gir et proxemie ou... la réappropration de IEspace-Temps naturel, Loisir et Socibte, vol, 8, no 1, 1985, pp. 217-250.

PINEAU, Gaston el collaborateurs, De l'wir, essai sum Fécoformation, chapitre de J-C. Jay-Payon: Le plein air, $P D$. 115-143, préface de madame Gro Hartem Brundiland, Paideia, Editions Sciences et Culture linc, 1992, $269 \mathrm{p}$.

POPCORN, Faith, Le rapport Popcorn - Comment vivrons-nous I'an 2000?, Les Editions de PHomme, 1994, 265 p

SOLOMON, Henry, Le sport ha tout prix, le mythe de I'exercice physique, 1985, $224 \mathrm{p}$.

\section{NOTES}

(1) Les principaux parcs doivent leurs origines aux monuments naturels et non... aux écosystemes donc, au spectacle naturel

(2) Le droit al la nature a été particulièrement présent au sein du national-socialisme allemand des annees 1930-1935.

(3) Le maintien de tous les 6osystemesn'est pas un concept absolu quand on considere que $90 \%$ des ecosystemes originaux sont naturellement disparus pour ètre remplaces par de nouveaux, en plus ou moins grands nombres. René Dubos

(4) Se rot ther a un texte du même auteur intitule: La valeur economique du patrimoine - environne. ment de 1994.

(5) II ne semble malheureusement pas qu'un tel typa de planification territoriale existe au Québec.

(6) En ce sens, les Etats-Unis semblent convaincus que les parcs sont... a tout le monde et non seulement le privilege de certains pratiquants punstes.

(7) Exemple de nature humanisée floristique insuffisamment développée au Québec. Pénune de jardins floraux de ce type.

(B) Cette demiere phrase s'appuie sur plusieurs anecdotes qui en disent long sur l'état d'esprit d'une époque. Au cours de la décennie 1980-1990, le directeur des parcs, alors en poste au Gouverne. ment, clamait que: "... de son vivant, il n y aurait amais une machine a coke dans les pares, parce que cela faisait américain, urbain et... capitaliste! Son predécesseur avait lui déslaré: *, ., qu'il fallait construire des arches à l'entrée des parcs of réserves, pour taire courber la téte au visiteur et ainsi l'inciter au respect de la naturea! 